\title{
Neurophysiological Biomarkers of Persistent Post-concussive Symptoms: A Scoping Review
}

\begin{abstract}
Sepehr Mortaheb 1,2,3, Maria Maddalena Filippini 1,2,4, Jean-François Kaux ${ }^{5}$, Jitka Annen ${ }^{1,2}$, Nicolas Lejeune ${ }^{1,2,6}$, Géraldine Martens ${ }^{1,5}$, Maria Antonia Fuentes Calderón ${ }^{7}$, Steven Laureys ${ }^{1,2}$ and Aurore Thibaut ${ }^{1,2 *}$

${ }^{1}$ Coma Science Group, GIGA-Consciousness, University of Liège, Liège, Belgium, ${ }^{2}$ Brain Clinic, University Hospital of Liège, Liège, Belgium, ${ }^{3}$ Physiology of Cognition Lab., G/GA-Consciousness, University of Liège, Liège, Belgium, ${ }^{4}$ Neuromotor and Rehabilitation Department, Azienda Unita Sanitaria Locale-Istituto di Ricovero e Cura a Carattere Scientifico (USL-IRCSS) di Reggio Emilia, Reggio Emilia, Italy, ${ }^{5}$ Physical Medicine and Sport Traumatology Department, Sports, FIFA Medical Centre of Excellence, IOC Research Centre for Prevention of Injury and Protection of Athletes Health, FIMS Collaborative Centre of Sport Medicine, University and University Hospital of Liège, Liège, Belgium, ${ }^{6}$ Institute of NeuroScience, University of Louvain, Brussels, Belgium, ${ }^{7}$ Neurorehabilitation (NEURORHB) Servicio de Neurorrehabilitación de Hospitales Vithas, Valencia, Spain
\end{abstract}

Background and Objectives: Persistent post-concussive symptoms (PCS) consist of neurologic and psychological complaints persisting after a mild traumatic brain injury (mTBI). It affects up to $50 \%$ of mTBI patients, may cause long-term disability, and reduce patients' quality of life. The aim of this review was to examine the possible use of different neuroimaging modalities in PCS.

Methods: Articles from Pubmed database were screened to extract studies that investigated the relationship between any neuroimaging features and symptoms of PCS. Descriptive statistics were applied to report the results.

Results: A total of 80 out of 939 papers were included in the final review. Ten examined conventional MRI (30\% positive finding), 24 examined diffusion weighted imaging (54.17\% positive finding), 23 examined functional MRI (82.61\% positive finding), nine examined electro(magneto)encephalography (77.78\% positive finding), and 14 examined other techniques (71\% positive finding).

Conclusion: MRI was the most widely used technique, while functional techniques seem to be the most sensitive tools to evaluate PCS. The common functional patterns associated with symptoms of PCS were a decreased anti-correlation between the default mode network and the task positive network and reduced brain activity in specific areas (most often in the prefrontal cortex).

Significance: Our findings highlight the importance to use functional approaches which demonstrated a functional alteration in brain connectivity and activity in most studies assessing PCS.

Keywords: neuroimaging, persistent post-concussive symptoms, diffusion weighted imaging, functional MRI, electroencephalography, mTBI 


\section{INTRODUCTION}

Emerging evidence shows that the long-term consequences of concussion-also known as mild traumatic brain injury (mTBI) - can be broad and last long (1). Those consequences can last for a few minutes (e.g., dizziness and loss of consciousness), for a few hours (e.g., post-traumatic amnesia, headaches, and attentional and/or sensitivity issues), or for weeks or months (e.g., fatigue, irritability, anxiety, and insomnia), which are known as persistent post-concussive symptoms (PCS) and can have an important socio-economic impact (2). Up to $50 \%$ of patients who had a concussion suffer from PCS at 3 months post injury (3). One study highlighted that $27 \%$ of patients who had a concussion could not return to their previous work 12 months post-injury; furthermore, among the patients who return to their previous work, 84\% still reported PCSrelated complaints (4). These authors also showed that, besides demographics predictors (e.g., age and education) and injury characteristics (e.g., cause and severity of injury), the indicators of psychological distress and employment were of influence on work resumption. Proper diagnosis of a concussion is the first step to an accurate disease management which can further enable the re-employment of subjects and can reduce economic burden on the society. Lack of knowledge (e.g., inaccurate belief that the concussion is systematically linked to loss of consciousness) and unfamiliarity with common guidelines in sports teams (despite the increasing interest of international federations in establishing new guidelines and regulations) are also a serious issue which limit proper diagnosis and the appropriate management of patients who suffered from mTBI $(5,6)$.

The heterogeneity of injury and symptoms, the current limitations in the sensitivity of imaging, and the lack of sensitivity of classical biological markers represent important challenges for

\footnotetext{
Abbreviations: ACC, anterior cingulate cortex; MKT, mean kurtosis tensor; $\mathrm{AD}$, axial diffusitivity; MMN, mismatch negativity; AI, axonal injury; MPFC, medial prefrontal cortex; BC-PSI, British Columbia Postconcussion Symptom Inventory; MRS, magnetic resonance spectroscopy; BOLD, blood-oxygen-level dependent; mTBI, mild traumatic brain injury; CT, computed tomography; OFC, orbitofrontal cortex; DAN, dorsal attentional network; PCC, posterior cingulate cortex; DMN, default mode network; PCD, post-concussion disorder; DLPFC, dorsolateral prefrontal cortex; PCS, post-concussion syndrome; DSMIV, Diagnostic and Statistical Manual for Mental Disorders-IVth Edition; PCS, post-concussion syndrome negative; DTI, diffusion tensor imaging; PCS+, post-concussion syndrome positive; DWI, diffusion-weighted imaging; PCSNIM, PCS-negative impression management; EEG, electroencephalography; PCSQ, Post-concussive Symptom Questionnaire; ERF, event-related field; PET, positron emission tomography; ERP, event-related potential; PO, poor outcome; FA, fractional anisotropy; PTSD, post-traumatic stress disorder; FC, functional connectivity; RD, radial diffusitivity; FLAIR, fluid-attenuation inversion recovery; ROI, region of interest; fMRI, functional magnetic resonance imaging; RPQ, Rivermead Post-concussion Symptoms Questionnaire; fNIRS, functional nearinfrared spectroscopy; RSN, resting-state network; FPC, fronto-parietal cortex; SCAT, Sport Concussion Assessment Tool; GM, gray matter; SN, salience network; GO, good outcome; SP, shortest path; ICBs, intracranial bleeds; SPECT, single photon emission computed tomography; ICD-10, International Classification of Diseases-10th Edition; SWI, susceptibility-weighted imaging; IFO, inferior fronto-occipital fasciculus; TBI, traumatic brain injury; ImPACT, immediate post-concussion assessment and cognitive testing; TPN, task positive network; MD, mean diffusitivity; vmPFC, ventromedial prefrontal cortex; MEG, magnetoencephalography; WM, white matter.
}

the development of diagnostic tools and biomarkers of good and poor recovery. Acute conventional computed tomography (CT) findings do not correlate with long-term outcomes such as PCS $(7,8)$. An extensive meta-analysis suggests that $\mathrm{CT}$ should only be performed under certain circumstances, such that when patients are at risk of severe intracranial injuries and present specific symptoms (e.g., loss of consciousness for more than $5 \mathrm{~min}$, declining in neurological status, or having seizures among others) (9). Besides CT, other techniques offer alternatives to study brain structural and functional integrity, such as MRI [conventional MRI, functional MRI (fMRI), diffusion-weighted imaging (DWI), magnetic resonance spectroscopy (MRS)], singlephoton emission computed tomography (SPECT), positron emission tomography (PET), magnetoencephalography (MEG), electroencephalography (EEG), or functional near-infrared spectroscopy (fNIRS). Neuroimaging techniques have been shown to be sensitive biomarkers for an accurate diagnosis and prognosis of PCS. Importantly, damage to the structural integrity (e.g., alterations of anisotropy and diffusion measures of the white matter) and disrupted functional network communication (e.g., alterations in resting-state functional network connectivity or in regional activations while performing a cognitive task) in the acute and subacute phases of mTBI are the main factors which lead to PCS (10). While these biomarkers are sufficiently sensitive to detect concussion-related symptoms, there are also some inconsistencies across studies about the way these markers are related to the concussion (11). As a result, more studies and reviews are needed for a comprehensive understanding of PCS and how neuroimaging biomarkers can help to diagnose and prognose such syndrome.

Taking all these into account, our review aims to address which neuroimaging features of mTBI are relevant to the clinical expression of PCS and could therefore potentially be used as biomarkers for PCS diagnosis and/or prognosis whether crosssectionally or longitudinally.

\section{MATERIALS AND METHODS}

A search on PubMed database was performed on June 13, 2018 and updated on May 14, 2020, which included the following terms: mTBI, mild traumatic brain injury, post-concussive syndrome, post-concussion syndrome, and neuroimaging, magnetic resonance imaging, functional magnetic resonance imaging, diffusion tensor imaging, diffusion spectrum imaging, diffusion-weighted imaging, susceptibilityweighted imaging, diffusion kurtosis imaging, positron emission tomography, computed tomography, single-photon emission computed tomography, magnetoencephalography, electroencephalography, near-infrared spectroscopy, functional near-infrared spectroscopy, resting state, and functional connectivity.

The inclusion criteria were as follows: (1) articles published in the last 10 years (from the time of database search, which leads to articles published from 2008), (2) in humans (adults: 16+ years old), (3) on post-concussion syndrome, (4) patients had to be 


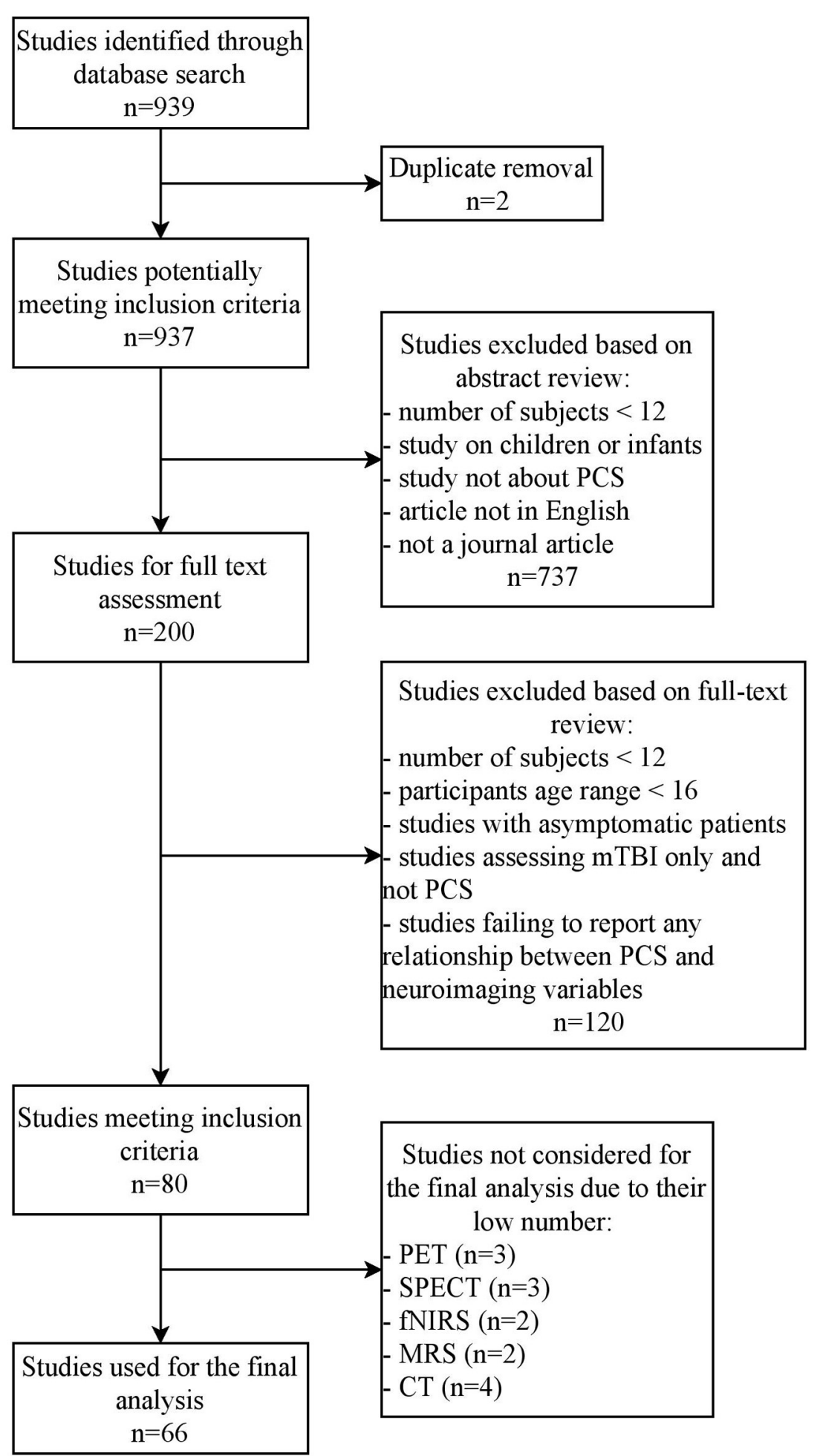

FIGURE 1 | PRISMA flowchart. A search over the Pubmed database yielded 939 studies, after which they were screened in the first round based on their abstracts and in the second round based on the main text of the remaining studies (PET, Positron Emission Tomography; SPECT, Single Photon Emission Computed Tomography; fNIRS, Functional Near Infrared Spectroscopy; MRS, Magnetic Resonance Spectroscopy; CT, Computed Tomography). 


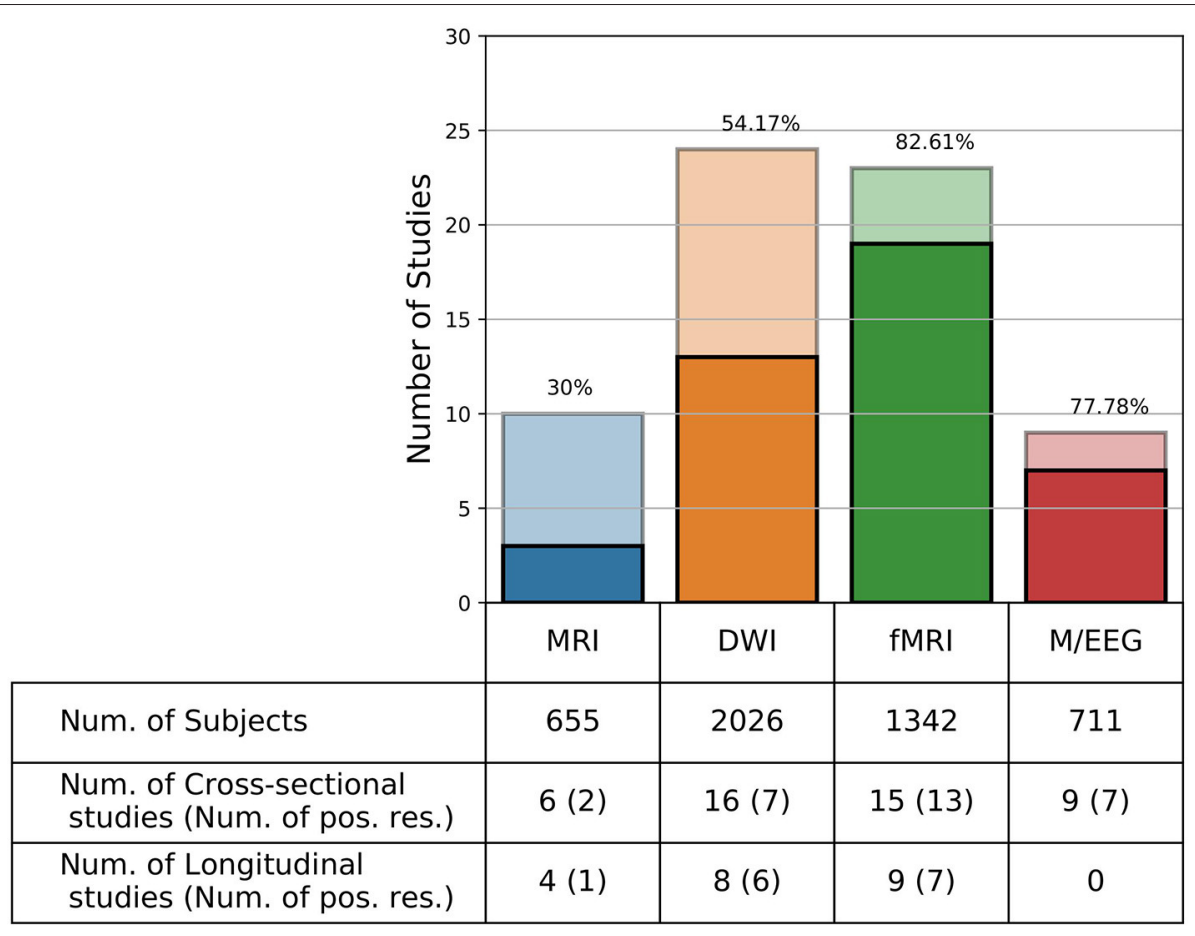

FIGURE 2 | Number of studies per imaging technique. Number of studies (Y-axis) per imaging technique (X-axis). Functional MRI has the highest number of experiments with positive results (i.e., studies which found an association between neuroimaging features and post-concussive symptoms). Darker colors show the number of studies with positive results, while lighter colors show the studies without significant findings. The number above each bar represent the percentage of studies with positive results.

assessed with one of the following techniques: CT, MRI, PET, SPECT, MEG, NIRS, or EEG, and (5) published in English.

The exclusion criteria were as follows: (1) articles including $<12$ subjects to exclude case studies and pilot studies [based on the criteria discussed in Julious (12)] and (2) reviews, opinion papers, or case reports.

Our outcome measures involved the association between neuroimaging and/or neurophysiological biomarkers and the presence or severity of PCS symptoms as measured cross-sectionally or longitudinally with the following scales (non-exhaustive list): Post-concussion Symptom Scale, Postconcussive Symptom Questionnaire, PCS-19, PCS-Negative Impression Management Scale, Sport Concussion Assessment Tool (all versions), International Classification of Diseases-10th edition Diagnostic Criteria for Post-concussion Syndrome, Diagnostic and Statistical Manual for Mental Disorders-IVth edition Diagnostic Criteria for Post-concussional Disorder, Rivermead Post-concussion Symptoms Questionnaire, British Columbia Post-concussion Symptom Inventory, Neurobehavioral Symptom Inventory, and Immediate Postconcussion Assessment and Cognitive Testing. In order to prevent differences in brain development and because some of the mentioned behavioral scales are not validated for adolescents, we limited our review to the studies with subjects older than 16 years old.

Two independent blinded reviewers (SM and MF) first reviewed the abstracts for inclusion using the Rayyan software
(13). In case of disagreement, a third reviewer (AT) was involved in the final decision. Descriptive statistics were used to report the results. In this review, "positive results" refer to the studies for which we found a statistically significant association between at least one neuroimaging marker and PCS.

\section{RESULTS}

The search yielded 939 articles, from which 859 were excluded and 80 articles were kept for a final review (see Figure 1). The imaging techniques used in these 80 studies were conventional MRI (10 papers), DWI (24 papers), functional MRI (23 papers), EEG/MEG (nine papers), PET (three papers), SPECT (three papers), fNIRS (two papers), MRS (two papers), and CT (four papers).

We here present the results for conventional MRI, DWI, fMRI, and EEG/MEG (66 papers; see Figure 2 and Table 1; for the definitions of some specific outcome measures mentioned in this table, see Table 2). For the other techniques, only a couple of articles were found $(n=14)$, which limits the generalizability of the results.

In general, out of the above-mentioned 66 papers, $42(63.63 \%)$ reported positive results, $45(68.18 \%)$ performed a cross-sectional study, $20(30.30 \%)$ performed a longitudinal study, and one $(1.51 \%)$ performed both kinds of analysis. Among the total of 
TABLE 1 | Studies included in the final review, sorted by neuroimaging technique.

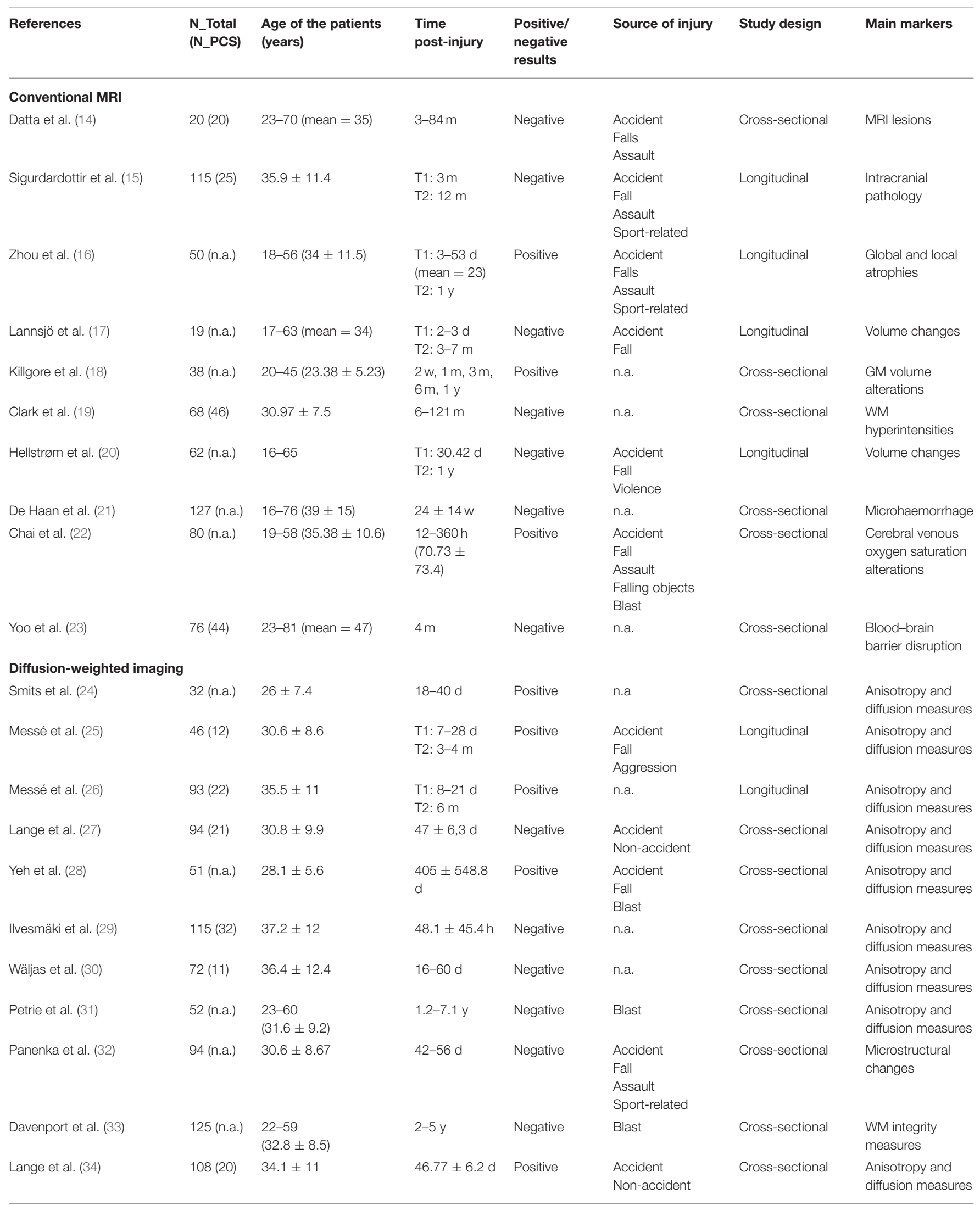


TABLE 1 | Continued

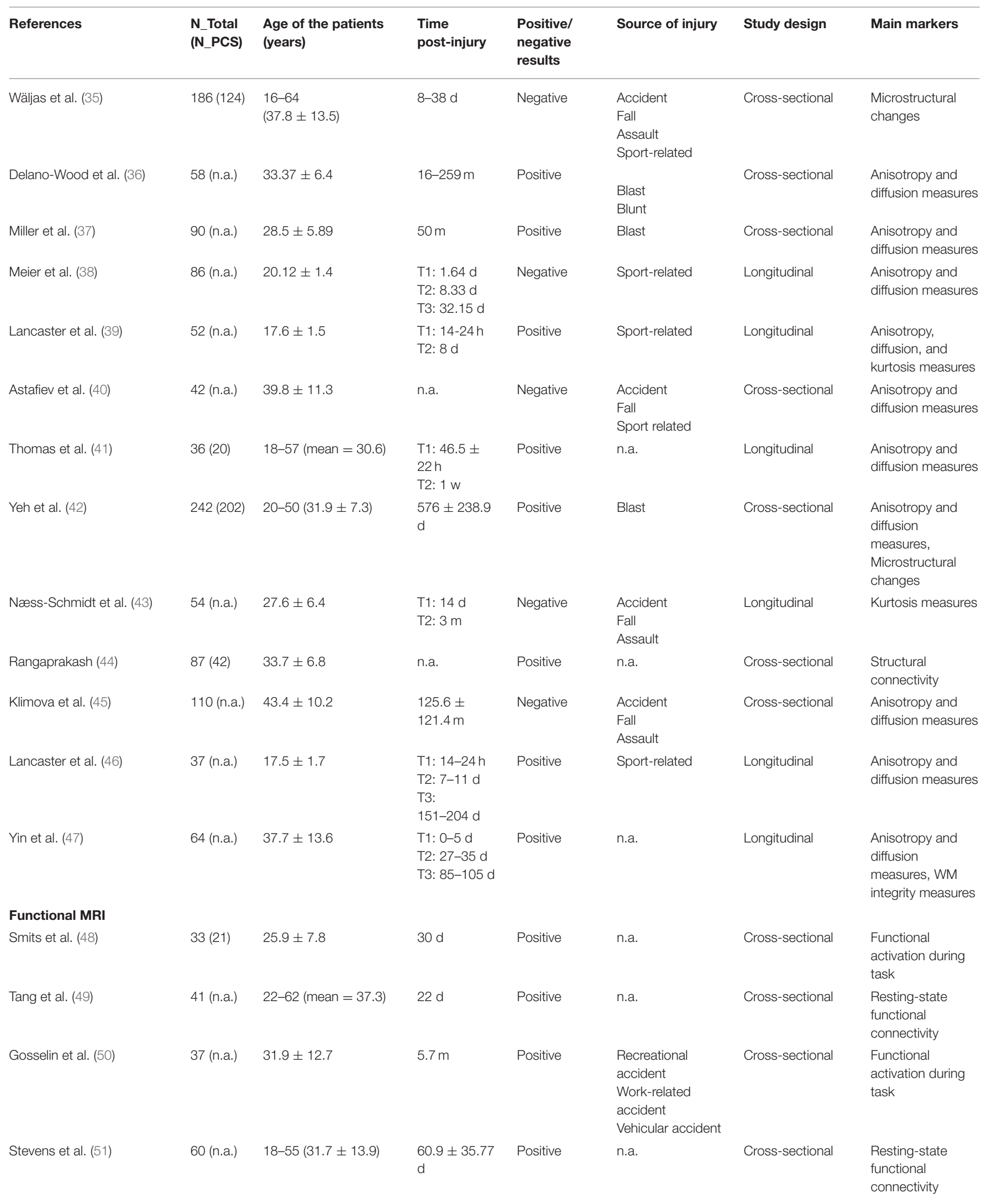


TABLE 1 | Continued

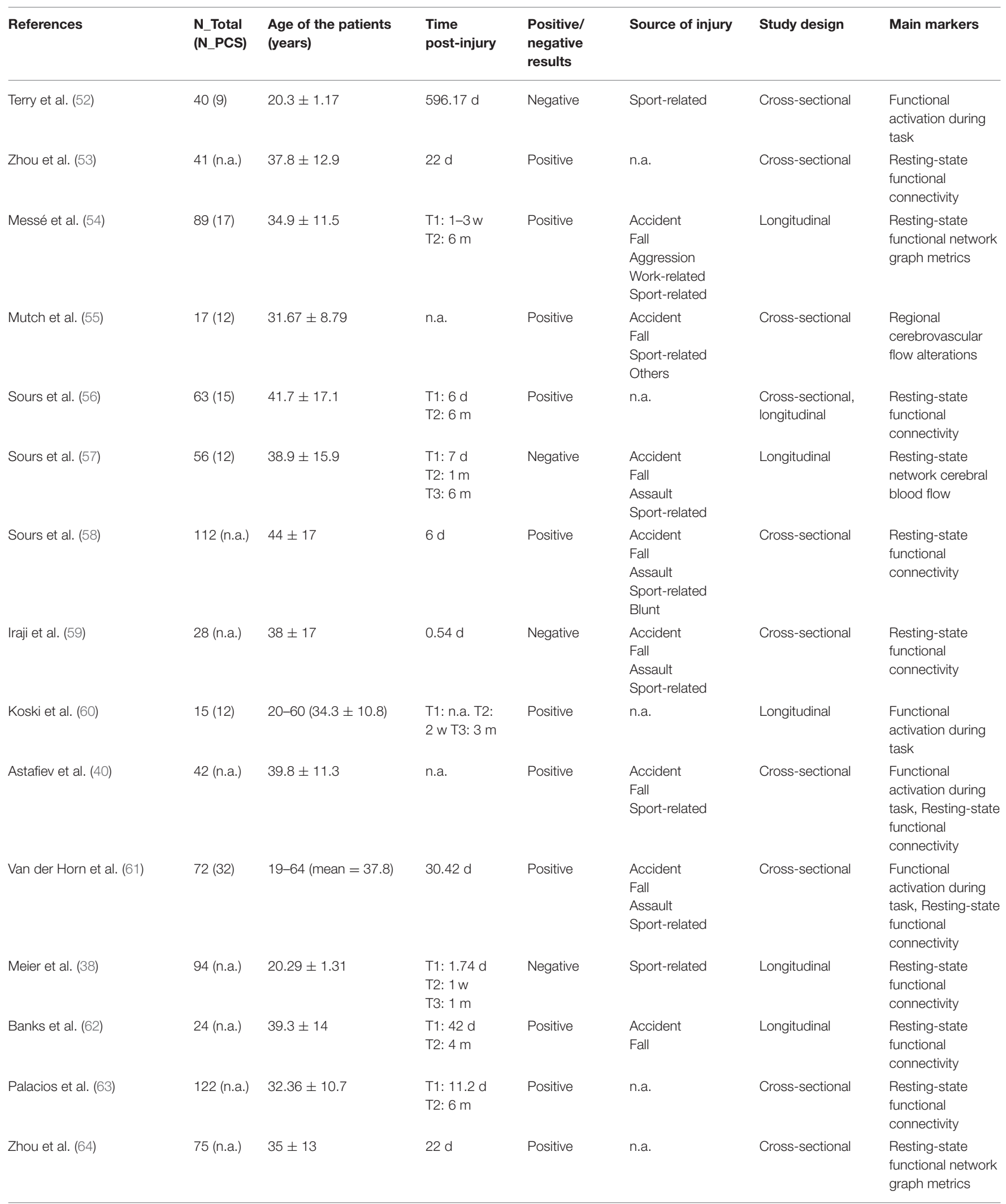


TABLE 1 | Continued

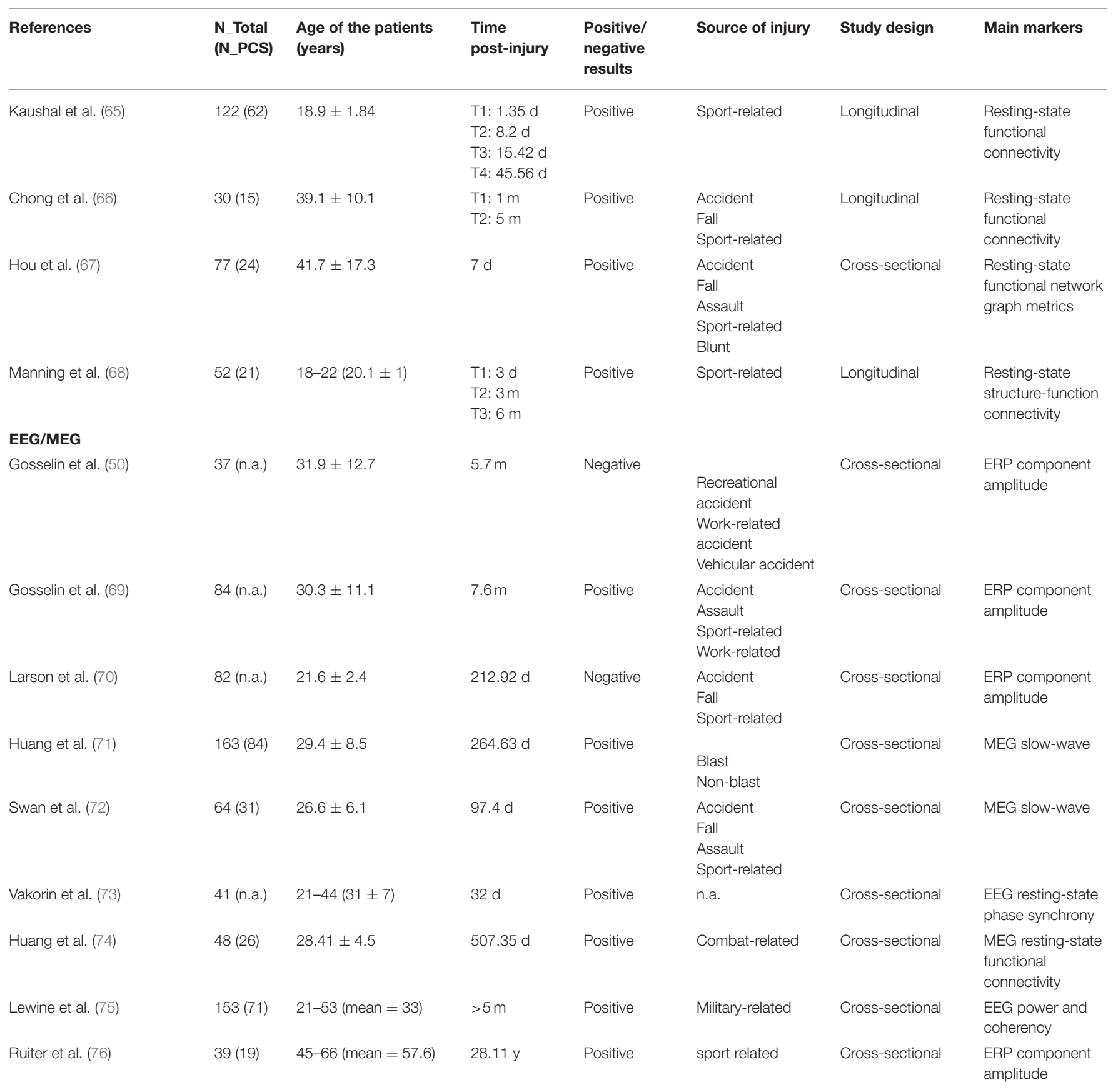

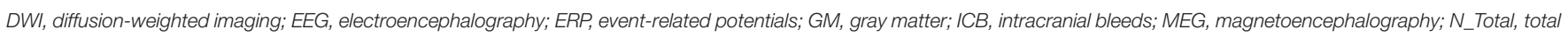

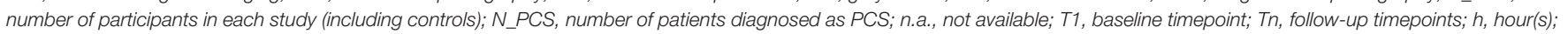
d, day(s); w, week(s); m, month(s); y, year(s); WM, white matter.

46 cross-sectional studies, 29 (63.04\%) reported positive results, while among the total of 21 longitudinal studies, 14 (66.67\%) also reported positive results. Considering all these studies, a total number of 4,734 subjects (including controls) underwent neuroimaging, with the majority (2,026 participants) being studied using DWI. The main findings are summarized below based on the imaging technique, and more extensive results are presented in Table $\mathbf{1 .}$

\section{Conventional MRI}

Among the 10 studies retrieved (655 participants), three showed positive results (30\%), six (60\%) were performed cross-sectionally (two with positive results), and four (40\%) were performed longitudinally (one with positive results). The main results consist of a smaller volume of cingulate gyrus isthmus, ventromedial prefrontal cortex (vmPFC), and fusiform gyrus. 
TABLE 2 | Definition of specific outcome measures in the reviewed papers.

\begin{tabular}{ll}
\hline Explanation Outcome measure \\
\hline
\end{tabular}

\section{Diffusion-weighted imaging parameters}

In diffusion tensor imaging, we can model water molecule diffusion by tensors. The three main eigenvalues and eigenvectors of tensor representation can give information about the main diffusion direction. In the following definitions, consider $\lambda_{1}$ as the maximum eigenvalue (main diffusion direction) and $\lambda_{2}$ and $\lambda_{3}$ as the two shortest eigenvalues (perpendicular to the main diffusion direction).

Axial kurtosis

Diffusion kurtosis imaging is a diffusion technique based on the non-Gaussian diffusion of water. It characterizes non-Gaussian diffusion by estimating the excess kurtosis of the displacement distribution and gives an idea of the underlying tissue complexity. We can perform a kurtosis tensor estimation of the water molecule diffusion data like the diffusion tensor estimation technique.

\section{Graph analysis parameters}

If we represent a network as a graph, different mathematical features can be extracted from the graph which represents the topological characteristics of the network.
Radial diffusivity (RD)

Mean diffusivity (MD)

Fractional anisotropy

Axial diffusivity $(A D)$

$\mathrm{AD}=\lambda_{1}$

It is only sensitive to diffusion in the longest eigenvalue direction. Highly organized structures like white matter pathways and large open cavities like ventricles have generally high levels of diffusion and are sensitive to this measure.

$\mathrm{RD}=\left(\lambda_{2}+\lambda_{3}\right) / 2$

It represents the two shortest eigenvalues and shows low values in highly organized and dense structures like white matter pathways, intermediate values in gray matter, and high values in regions with cerebral spinal fluid (CSF)

$\mathrm{MD}=\left(\lambda_{1}+\lambda_{2}+\lambda_{3}\right) / 3$.

It is specifically sensitive to CSF, which has high values of average diffusion.

It provides the relative difference between the largest eigenvalue as compared to the others; it quantifies the fraction of diffusion that is anisotropic. This leads to selective high values in white matter, but not gray matter or CSF.

The kurtosis value along the largest eigenvalue direction of the diffusion ellipsoid.
Mean kurtosis tensor

Bilateral thalamic resting-state network (RSN) degree of symmetry

Local efficiency

Modularity

Relative betweenness centrality

Clustering coefficient

Minimum spanning tree (MST)

Average shortest path
It quantifies the degree of deviation from Gaussian diffusion and is based only on the trace of the kurtosis tensor.

The percentage of the total voxel number of bilateral thalamic RSNs that correlated with not only the left but also the right thalamus.

The local efficiency of a node characterizes how well information is exchanged by its neighbors when this node is removed.

The strength of division of a network into modules.

Betweenness centrality is a measure of the influence of a vertex over the flow of information between every pair of vertices under the assumption that information primarily flows over the shortest paths between them. The betweenness centrality increases with the number of vertices in the network, so a normalized version is often considered with the centrality values scaled between 0 and 1.

It is a measure of the degree to which nodes in a graph tend to cluster together.

It is a subgraph containing all vertices of the main graph without any loop and with minimum total weights. The total weight of the MST of a sample graph shows the minimal broadcast cost of the main graph.

It is also called characteristic path length, is a measure of global connectivity, and shows the efficiency of information exchange in a network. The lesser values show a higher efficiency. 
TABLE 2 | Continued

\begin{tabular}{|c|c|c|}
\hline Explanation & Outcome measure & Outcome measure definition \\
\hline \multicolumn{3}{|l|}{ ERP components } \\
\hline \multirow{4}{*}{$\begin{array}{l}\text { Every ERP signal is characterized by peaks and troughs } \\
\text { after stimulation time, which are called the ERP } \\
\text { components. Although some components have specific } \\
\text { names, others are named based on their polarity (P for } \\
\text { positive peaks and } N \text { for negative ones) and the time of } \\
\text { their appearance after stimulation (in millisecond). } \\
\text { Sometimes, instead of their appearance time, their } \\
\text { appearance order is stated }\end{array}$} & P300 & $\begin{array}{l}\text { A positive peak being observed around } 300 \mathrm{~ms} \text { after } \\
\text { stimulus time and is mainly related to the consciously } \\
\text { controlled attention. }\end{array}$ \\
\hline & N350 & $\begin{array}{l}\text { A negative peak being observed around } 350 \mathrm{~ms} \text { after } \\
\text { stimulus onset and is mainly related to cognitive } \\
\text { processing during sleep onset period. }\end{array}$ \\
\hline & N1 & $\begin{array}{l}\text { A negative peak being observed around } 150-200 \mathrm{~ms} \\
\text { after the stimulus and is mainly related to auditory } \\
\text { attention. }\end{array}$ \\
\hline & Mismatch negativity & $\begin{array}{l}\text { A negative peak around } 150-250 \text { ms post-stimulus } \\
\text { which is related to pre-attentive processing independent } \\
\text { of conscious attention. }\end{array}$ \\
\hline
\end{tabular}

\section{Diffusion-Weighted Imaging}

Among 24 studies retrieved (2,026 participants), 13 showed positive results $(54.17 \%), 16(66.67 \%)$ were performed crosssectionally (seven with positive results), and eight (33.3\%) were performed longitudinally (six with positive results). The main results consist of alterations of anisotropy and diffusion measures mainly in the corpus callosum, longitudinal fasciculi, and tracts in the internal capsule.

\section{Functional MRI}

Among 23 studies retrieved (1,342 participants), 19 showed positive results $(82.61 \%), 14(60.87 \%)$ were performed cross-sectionally (12 with positive results), while eight $(34.78 \%)$ were performed longitudinally (six with positive results), and one (4.35\%) was performed in both types. Seventeen $(73.91 \%)$ studies used resting-state paradigm for data acquisition, four (17.39\%) used cognitive tasks, and two $(8.70 \%)$ used both paradigms. Out of 19 resting-state studies, $16(84.21 \%)$ reported positive results, and out of six task-based studies, five $(83.33 \%)$ also reported positive results. The main results consist of alterations of the anticorrelation between the default mode network and the task postive network, which is present in healthy subjects, and an abnormal increase of thalamo-cortical connectivity (see Figure 3).

\section{Electro(magneto)encephalography}

Among nine studies retrieved (five EEG and four MEG; 711 participants), seven showed positive results (three EEG and four MEG; 77.78\%), and all nine studies (100\%) were performed cross-sectionally (seven with positive results). The main results consist of a reduction of event-related potentials (ERP) components, such as P300, N350, N2b, and N1, and mismatch negativity, an increase of relative theta power, a decrease of relative alpha power, a decrease of beta band interhemispheric coherency, and an abnormal slow-wave activity in orbito-frontal cortex, vmPFC, and fusiform gyrus.

\section{DISCUSSION}

This article aims to review the current state of the art in neuroimaging and neurophysiological techniques for the diagnosis of PCS. Out of 80 papers studied in this scoping review, the majority (66 articles) were performed using structural/functional MRI and EEG/MEG, which were conducted either cross-sectionally or longitudinally. In this review, we found that the percentage of positive results is almost the same for both types of study design. However, we should note that the number of cross-sectional studies was greater than twice the number of the longitudinal ones (46 articles vs. 21 articles). Therefore, in order to have a wider overview of the progress of PCS and the biomarkers which can correlate with it more longitudinal studies are needed. Considering the imaging modality, most positive findings were found among studies using functional approaches (i.e., fMRI and EEG/MEG). However, the number of studies using electrophysiological techniques (i.e., EEG) remains limited compared to neuroimaging studies (i.e., fMRI), while the former is more practical and more available in clinical practice. The main findings per technique are discussed below respectively for conventional MRI, DWI, fMRI, and EEG/MEG.

\section{Conventional MRI and DWI}

Considering both conventional MRI and DWI studies, only $47 \%$ of the structural MRI studies identified specific abnormalities in patients with PCS. Although DWI studies showed more positive findings compared to conventional MRI (54 vs. 30\%), 


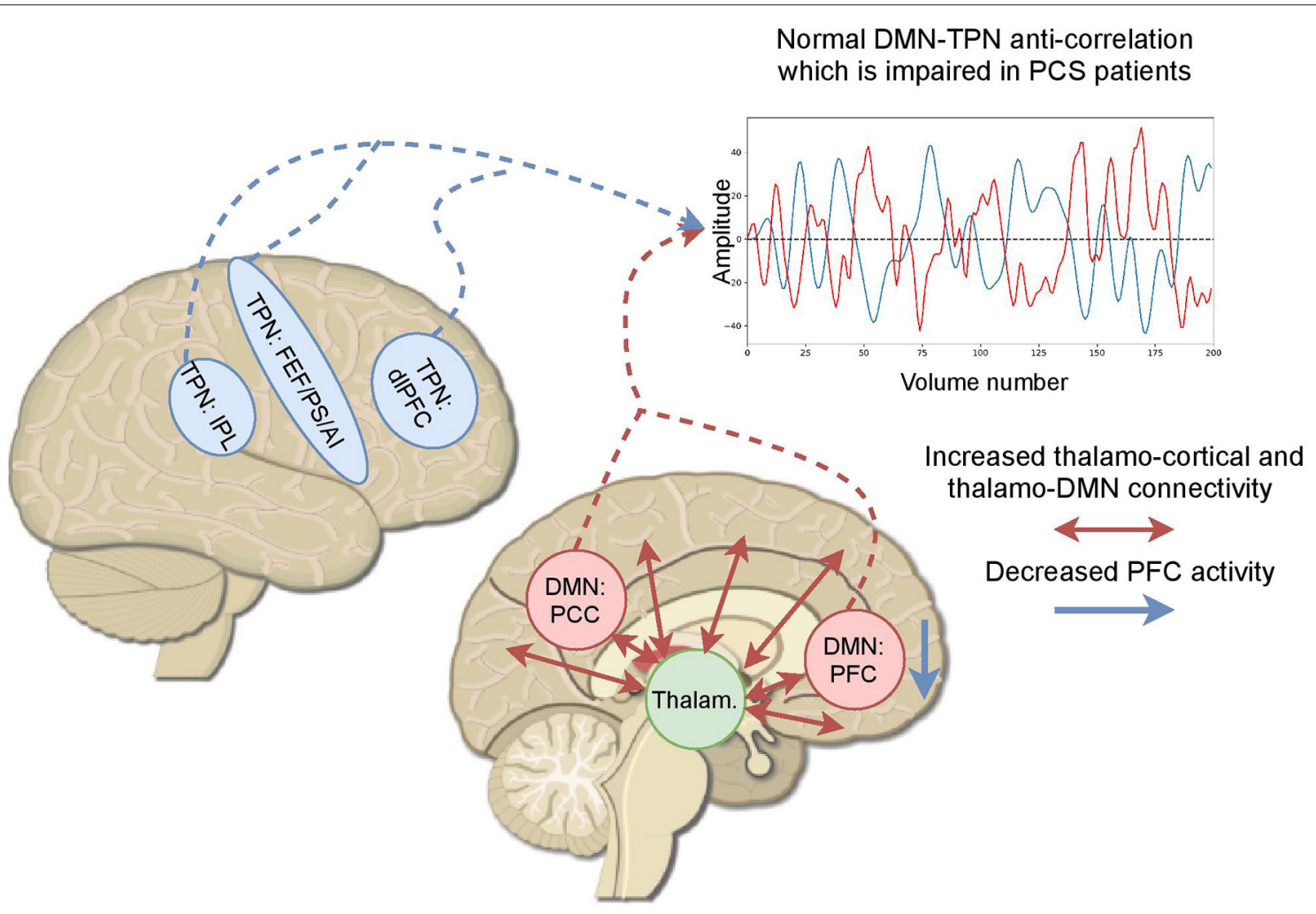

FIGURE 3 | Based on the fMRI studies exploring post-concussive symptoms (PCS), the main findings can be summarized as the abnormal increase of thalamo-cortical connectivity, alterations in the anticorrelation of the default mode network and the task positive network, and a reduction of the activity in the prefrontal cortex. A time series shows a created example of a normal DMN-TPN anticorrelation which becomes altered in patients with PCS. Al, anterior insula; dIPFC, dorsolateral prefrontal cortex; DMN, default mode network; FEF, frontal eye fields; IPL, inferior parietal lobe; PCC, posterior cingulate cortex; PFC, prefrontal cortex; PS, precentral sulcus; TPN, task-positive network.

they still have low positive findings compared to functional MRI acquisitions (54 vs. $83 \%$ ). However, we should notice that, in clinical practices, the acquisition of good-quality DWI images is much harder than acquiring good-quality MRI due to the high sensitivity of DWI images to motion. Structural MRI allows one to evaluate anatomical and structural brain damage in a qualitative or quantitative way using different acquisition sequences-for example, a conventional T1-weighted sequence is suitable to quantify and analyze changes in the volume and thickness of different brain regions. It is known that brain volume loss (whether globally or regionally) is a consequence of moderate or severe brain injuries (16) - for example, the anterior cingulate and left cingulate gyrus isthmus in the white matter are among the regions which show atrophy over time after a brain injury (16). Because the anterior parts of the cingulum play vital roles in different cognitive systems [e.g., working memory; (77)], atrophy in this area can explain the persistent symptoms after the concussion. The gray matter volume within vmPFC and the fusiform gyrus was also found to positively correlate with PCS symptoms, specifically emotional functioning (18). This may be due to the fact that the vmPFC has a strong connection with the amygdala and is involved in self-control and decision making (78).
A widely used MRI sequence to study the structural changes of the brain after PCS is DWI, which is mainly used to study changes of the white matter tracts. To analyze these tracts quantitatively, four main parameters of fractional anisotropy, axial diffusivity, radial diffusivity, and mean diffusivity were explored. The PCS-associated white matter abnormalities can be mainly characterized by a decrease in FA values and an increase in $\mathrm{MD}, \mathrm{AD}$, and $\mathrm{RD}$ values, respectively, in the injured tracts (FA and MD were the most commonly reported parameters to be altered compared to the others). The main reported tracts with axonal injury in PCS patients were the corpus callosum $(24-26,28,34,47)$, the longitudinal fasciculi $(24-26,34,42)$, and tracts in the internal capsule $(24,26,28,34,47)$. As these tracts are the main pathways of widespread cortical and subcortical connections, any axonal damage in these tracts can lead to various cognitive symptoms in patients with PCS. These axonal damages are mainly due to the rotational acceleration and/or deceleration forces happening when the concussion occurs (24). In addition to these main tracts, altered measures of anisotropy and diffusivity were also reported in the frontooccipital fasciculi $(24,25,47)$, the uncinate fasciculus $(24,41,42)$, the cortico-spinal tract (25), the thalamic radiation $(25,28,34$, 42 ), the cingulum bundle (28), and the pontine tegmentum 
(36). By applying tractography algorithms on the DWI images, structural connectivity can also be analyzed between different cortical regions. Using this technique, it has been shown that, compared to patients with post-traumatic stress disorder (PTSD) only, subjects with PCS and PTSD have a greater diversity in their structural connectivity between the hippocampus and the striatum, which could be associated with PCS but not PTSD (44).

In summary, the structural neuroimaging studies evaluating gray matter and white matter integrity found impairments of the cingulate gyrus and the prefrontal area, while for the white matter the corpus callosum, the longitudinal fasciculi, and internal capsule were the most frequently reported structures to be impaired or linked to PCS severity. As we can see and also highlighted by Miller et al. (37), the axonal damages in patients with PCS are spatially heterogeneous. This could be due to the heterogeneity of the studied populations (athletes vs. regular people vs. veterans) and the heterogeneity of the symptoms (e.g., stress, headache, sleep disturbances, and attentional issues).

\section{Functional MRI}

In fMRI studies, the results can be presented based on changes in BOLD activation level or based on functional connectivity among regions (i.e., network-based analysis). Furthermore, functional networks can be summarized by graph analysis metrics which create a mathematical framework to study brain network topology in normal and pathological states in more detail. We discuss the fMRI findings of PCS under these different categories.

\section{BOLD Activation Level Analysis}

Among the studies using this technique, most of them looked at working memory and selective attention deficits, which are the two main cognitive domains affected after mTBI (48). Based on previous studies, the brain areas which are involved in these cognitive domains are mainly the dorsolateral and the ventrolateral prefrontal cortex, the supplementary motor area, the premotor area, posterior parietal area, and the anterior cingulate cortex $(48,77,79)$. The activation level of these areas during tasks requiring a high cognitive load is correlated with PCS severity, showing increased attentional and short-term memory demands (48). Some studies also showed hyperactivation in the parahippocampal gyrus and the posterior cingulate cortex (PCC), which are mainly related to episodic memory and memory retrieval (48). The activation of these regions in high-load working memory and selective attention tasks shows a potential cerebral compensatory response to a possible microstructural injury in patients with PCS (48). Hyperactivity was also observed in the left angular gyrus, which resulted in the same behavioral accuracy for patients with PCS and healthy subjects in performing a working memory task (69). Taking all together, it can be stated that patients with PCS use more cognitive resources to perform an attention or working memory task with the same accuracy as the healthy subjects.

A decrease in BOLD activation level in the medial prefrontal cortex was also observed in patients with PCS $(50,61)$. It is known that this region is important for executive functioning and emotion regulation $(80,81)$. In addition, it is a core area of the default mode network (DMN) and an important relay station between DMN and other executive networks (61). As a result, impairment in this area can lead to serious cognitive and affective impairments $(50,61)$. Using transcranial magnetic stimulation as a treatment, an increase of the medial prefrontal cortex activation level during a working memory task was found and was associated with less severe PCS symptoms (60).

Besides working memory and selective attention, light sensitivity and headaches were also studied using a visual tracking task. A higher BOLD activity in the middle temporal/lateral occipital regions was observed compared to healthy subjects when the patients were exposed to visual stimuli. These regions are known as extra-striate visual regions involved in motion and object processing. The higher activation in these regions was also associated with higher FA values in the same region. In sum, light sensitivity and the associated headaches could be related to an abnormal sensitivity of motion-sensitive neurons (40).

\section{Functional Connectivity Analysis}

In a general view, abnormal functional connectivity can be found in almost all the functional networks which can be detected in the resting state (51). While few studies have reported an abnormal whole-brain hyperconnectivity especially in the acute phase (65), other studies have shown an overall whole-brain connectivity reduction in these patients $(56,63)$. Whole-brain connectivity analysis gives us a general view of the functional organization of the brain; however, focusing on specific networks or regions, chosen in a hypothesis-driven way, gives more insights about network configuration changes and a more fine-grained understanding of the pathology. Among different networks and regions, DMN and the thalamus were found to be the most affected ones.

\section{Default Mode Network}

The DMN is a network mainly related to internal awareness (82). Posterior regions of the DMN, such as the precuneus, showed more vulnerability to traumatic injury (53). A decrease of the connectivity of the precuneus inside the DMN and its increased connectivity with the primary and secondary visual networks and the left fronto-parietal network can alter the transition of attention from internal to external awareness (53). Another critical area in the DMN is the PCC. The decreased connectivity of the PCC within the DMN is associated with an increased connectivity of the medial prefrontal cortex (mPFC), which potentially shows the compensatory role of the mPFC to the impaired neurocognitive functions. Indeed the $\mathrm{mPFC}$ and the PCC are intrinsically interdependent inside the DMN, and their function is highly complementary (53). The anterior regions of the DMN, such as the anterior cingulate cortex (ACC), have also shown altered connectivity in these patients. The connectivity of the ACC with the emotional, the primary visual, and the higher-order processing networks is negatively correlated with the severity of the PCS (51). Considering the role of the ACC in pain processing, error and conflict detection, behavioral and cognitive control, as well as emotional processing, this could potentially explain the different complaints in mTBI patients. In addition to the role of the DMN in internal awareness, the interaction between the DMN and the task positive network (TPN) is crucial for performing executive functions. In fact, the balance between internal and external awareness is supported by 
the interaction of these two networks with temporally alternating higher activity states (83). In patients with PCS, the deactivation of the DMN is not strong enough while they are performing a working memory task (61). Specifically, an increase in the connectivity between the DMN and the TPN has been observed $(58,61,68)$. This altered anti-correlation between the DMN and the TPN (i.e., the lack of silencing of the DMN) could explain some of the difficulties in task performance that patients with PCS suffer from.

\section{Thalamus}

This subcortical hub plays an important role in memory, executive functions, attention, and emotion among others (81). Different studies have shown a PCS-related increase of the thalamo-cortical connectivity $(49,58,64)$ which can lead to an inefficient neuronal activity (64). The increased thalamocortical connectivity can be related to the alteration of the GABAergic inhibitory neurons in the thalamus $(49,58)$, resulting in a reduction of inhibitory control over the thalamo-cortical activity. The connectivity of the thalamus with other networks has also been studied. Evidence especially suggests that the connectivity between the thalamus and the DMN increases in patients with PCS $(58,62)$. As mentioned, a well-coordinated interaction between the DMN and the TPN is necessary for an appropriate balance between internal and external awareness and proper task execution. The fronto-parietal control (FPC) network (62) and the salience network (84) were suggested as networks that control this balance between the DMN and the TPN. The thalamus has connections with all of these networks and has a key role in this complex inter-network interaction. An increased connectivity between the thalamus and the DMN could lead to a malfunctioning of this modulatory interaction. In addition to the increased connectivity of both the thalamus and the DMN, a decrease of connectivity between the thalamus, the FPC, and the DAN was also reported (62). A normalization of this decreased connectivity was correlated with better PCS scores, while the connectivity with the DMN remained unchanged.

\section{Other Regions}

Besides the DMN and the thalamus, the reduction of homotopic functional connectivity in the pain processing regions is another finding which was shown to be normalized with symptom recovery and can serve as another potential biomarker of PCS (66).

\section{Graph Analysis}

One approach to study brain networks and their topological characteristics is the data-driven graph analysis. In this approach, connectivity measures are represented by weighted graphs [nodes represent regions of interest (ROIs), and edges represent connectivity between ROIs] - for example, graph modularity is increased in the temporal regions in the subacute phase and is decreased in the frontal regions in the chronic stage in patients with PCS (54). These changes can be localized mainly in regions related to executive functions, working memory, cognitive control, and attention. Some mathematical features of the graph (detailed in Table 2), such as the clustering coefficient, are also correlated with increased thalamo-cortical connectivity (64). Combining the graph analysis results of the functional networks with the diffusion-weighted images, it was revealed that regions of the brain showing a lower local relative betweenness centrality in the functional network have also lower fractional anisotropy; in addition, a decrease of global efficiency of the functional networks is related to diffuse axonal injury (64). The average shortest path weights and minimum spanning tree weights have also been shown to be increased in patients with PCS (67). Taking all these results together, the graph analysis shows that patients with PCS tend to have costly and less efficient network configurations for information transfer (67), which lead to cognitive impairments.

To summarize, the fact that positive findings were found for $82 \%$ of the papers showed that impairment in functional connectivity after mTBI could potentially explain most of the post-concussive symptoms. Mainly, besides a whole-brain connectivity reduction, alterations of the connectivity in the DMN and its interaction with other executive networks, especially alterations of the anti-correlation between the DMN and the TPN, could be associated with cognitive impairments. In addition, an increase of the thalamo-cortical connectivity due to alteration of GABAergic inhibitory neurons in the thalamus can also lead to difficulties in task performance. These functional impairments may recruit compensatory mechanisms in the brain and use extra cognitive resources while cognitive tasks are performed.

\section{Electro(magneto)encephalography}

In general, the EEG/MEG data can be analyzed whether in the sensor space (signals directly from surface sensors) or in the source space (i.e., signals of surface sensors are projected to the cortex to estimate the cortical location of the neural generators). Furthermore, various analysis methods can be performed on the standard frequency bands of the signals: delta $(0-4 \mathrm{~Hz})$, theta $(4-8 \mathrm{~Hz})$, alpha $(8-12 \mathrm{~Hz})$, beta $(12-30 \mathrm{~Hz})$, and gamma $(>30 \mathrm{~Hz})$. In addition, the EEG/MEG recordings can be done either in resting state or in response to stimuli, averaged over many trials, to measure ERP in case of EEG recording or event-related fields in case of MEG recording. Although in the analysis methods there are no serious differences between EEG and MEG signals in terms of clinical applicability and data acquisition, they are completely different. While EEG is widely available and can be performed easily in different clinical settings, MEG is scarcely available for clinical purposes. In the present review, eight out of 10 EEG/MEG studies identified neurophysiological changes linked to PCS (six EEG studies with four reporting positive results and four MEG studies all reporting positive results).

Considering ERP studies, they were mainly focused on the amplitude of P300 (69, 76), N350 (50, 69), N2b, N1, and mismatch negativity (MMN) components (76). All of them reported a reduction in the amplitude of such components. The first ERP study found that the amplitude of the N350 component in the frontal electrodes was decreased in patients with PCS while performing a working memory task compared 
to a control task $(50,69)$. Because this N350 amplitude reduction was correlated to the lower BOLD signal changes of the right middorsolateral prefrontal cortex (mid-DLPFC) in the same task, it is thought that this specific ERP component is related to the neural activity of the mid-DLPFC. In addition, the amplitude of P300 in parietal regions is decreased after a brain injury, and this reduction in amplitude is more significant when patients with PCS suffer from severe depressive symptoms (69). While both $\mathrm{N} 2 \mathrm{~b}$ and MMN are related to pre-attentive processing, MMN is observed independent of conscious attention, and $\mathrm{N} 2 \mathrm{~b}$ is observed in intentional conscious attentions (76). As a result, the reduction of amplitude of P300, N2b, and MMN can be linked to the attentional deficits which are common in PCS. The N1 component mainly reflects auditory attention and shows a reduced amplitude in case of auditory processing difficulties (76). In the analysis of resting-state EEG data, increase of relative theta power, decrease of relative alpha power, and decrease of beta band inter-hemispheric coherence have been observed (75).

Considering MEG recordings, it has been shown that the total MEG magnitude in the source space is correlated with the PCS scores (71). Slow-wave features are still more informative for cognitive impairment examination in this cohort of patients (72) - for example, the abnormal slow-wave activity in the orbitofrontal cortex and vmPFC has been shown to be associated with personality changes, concentration troubles, and emotional instability (71). Because these regions are highly connected to other cortical and subcortical areas, this abnormal activity can cause impairments in a vast domain of cognitive abilities (71). In addition, visual difficulties, such as blurred vision, have been explained by the abnormal slow-wave activity in the right fusiform gyrus (71), an important region for face, object, and body recognition and processing (85). The abnormal slow-wave generation in different regions has been associated with white matter abnormalities or micro-structure damages to the major tract in the same regions (86). The connectivity analysis features of MEG data in different frequency bands have also been shown to be informative biomarkers to study PCS after mTBI. Interregional resting-state phase synchrony in the alpha band could classify patients from healthy subjects with an accuracy of $80 \%$, in which the distance of each participant to the classification boundary was correlated with the symptom severity of that subject (73). The functional connectivity of ROIs in the prefrontal cortex, medial temporal lobe, putamen, and cerebellum with the whole brain was increased, while the functional connectivity of the right frontal pole with the rest of the brain in patients with PCS was decreased (74). These abnormal connectivity variations were observed in the beta, gamma, and low-frequency bands (i.e., $1-7 \mathrm{~Hz}$ ). The decrease of functional connectivity in the right frontal pole can be explained by diffuse axonal injury, which leads to the disruption of neural communications (87). On the other hand, the increase of functional connectivity in the prefrontal cortex, the medial temporal lobe, the putamen, and the cerebellum could be explained by the overexcitation of glutamate in the pyramidal neurons of the cortex, which may lead to an alteration of GABAergic interneurons (74).
Taking all these information together, different alterations in the EEG/MEG markers may be related to the white matter abnormalities and axonal injury, alterations of GABAergic neurons, and functional dysfunction of frontal regions, such as the DLPFC.

\section{Limitations}

Our work has some limitations that need to be taken into account. First of all, the heterogeneity of the studied populations resulted in the heterogeneity of the symptoms and the imaging markers. Secondly, a few number of studies explored PCS longitudinally. These two limitations made us unable to have specific strong prognostic recommendations for PCS. The third limitation was the few number of studies which used imaging techniques other than MRI and EEG. While imaging techniques like PET, SPECT, and fNIRS can provide more insight about the brain alterations in PCS. The few number of studies limited the generalization of their findings and therefore, were excluded from the analysis. Finally, most of the studies that we included in our review did not have a specific hypothesis, and the questions they were trying to answer were really diverse. This led to two main limitations of this review. First, we could not perform a meta-analysis which could help us to understand PCS more deeply. Second, because in the exploratory studies the number of biomarkers that the authors were planning to study is not known a priori, a comparison between the number of tested markers and the number of positive findings could not be performed in an appropriate and accurate way.

\section{CONCLUDING REMARKS}

In this scoping review, we studied the most reliable approaches to evaluate PCS and the common findings among studies which could explain PCS (i.e., biomarkers of PCS). Most of the studies using structural MRI to investigate the neural correlates of PCS did not find any specificities, except for an atrophy mainly involving the cingulate and the prefrontal areas linked to PCS symptoms, and abnormalities in the anisotropy and diffusion parameters of the corpus callosum, the longitudinal fasciculi, and the internal capsule. On the other hand, the majority of functional studies (fMRI and EEG/MEG) found an abnormal increase in the thalamo-cortical functional connectivity, which could be linked to the employment of compensatory mechanisms needed to perform cognitive tasks. In addition, an altered anticorrelation between the DMN and the TPN could explain some of the difficulties in task performance experienced by patients with PCS. Besides this, reduction of brain activity in specific areas was also found; the most frequently reported ones being the prefrontal cortex. The functional abnormalities in these above-mentioned brain regions and networks should draw the attention of physicians to provide appropriate care to these at-risk patients. The EEG/ERP studies identified a reduction in ERP component amplitudes related to attentional processes. However, only six studies used EEG, although compared to MRI or even MEG, this technique is easier to 
use in clinical practice. Further EEG studies could provide an insightful prediction of PCS outcome that could be more easily implemented in clinical practice. In addition, considering the heterogeneity of the results based on different neuroimaging modalities, it is recommended to conduct more multimodal studies. Furthermore, to decrease the amount of inconsistency of the results across studies, more longitudinal multi-center studies with bigger sample sizes are advised to be performed. It should be noted that, in most of the studies included in the review, no a priori or precise hypotheses were stated. We hope that our findings will guide authors to postulate specific hypotheses for future neuroimaging studies. So far, fMRI seems the most robust approach to study PCS severity, and future prospective longitudinal studies in a large sample of concussed patients using clinically relevant techniques such as EEG should determine the prognostic factors of good and poor outcome following a concussion.

\section{DATA AVAILABILITY STATEMENT}

The original contributions presented in the study are included in the article/Supplementary Material, further inquiries can be directed to the corresponding authors.

\section{REFERENCES}

1. Hiploylee C, Dufort PA, Davis HS, Wennberg RA, Tartaglia MC, Mikulis D, et al. Longitudinal study of postconcussion syndrome: not everyone recovers. J Neurotrauma. (2017) 34:1511-23. doi: 10.1089/neu.20 16.4677

2. Junn C, Bell KR, Shenouda C, Hoffman JM. Symptoms of concussion and comorbid disorders. Curr Pain Headache Rep. (2015) 19:46. doi: 10.1007/s11916-015-0519-7

3. Voormolen DC, Haagsma JA, Polinder S, Maas AIR, Steyerberg EW, Vulekovic $\mathrm{P}$, et al. Post-concussion symptoms in complicated vs uncomplicated mild traumatic brain injury patients at three and six months post-injury: results from the CENTER-TBI study. J Clin Med. (2019) 8:1921. doi: $10.3390 / \mathrm{jcm} 8111921$

4. Van der Naalt J, Van Zomeren AH, Sluiter WJ. One year outcome in mild to moderate head injury: the predictive value of acute injury characteristics related to complaints and return to work. Headache Quarterly. (1999) 10:158. doi: 10.1136/jnnp.66.2.207

5. McCrea M, Hammeke T, Olsen G, Leo P, Guskiewicz K. Unreported concussion in high school football players: implications for prevention. Clin J Sport Med. (2004) 14:13-7. doi: 10.1097/00042752-20040100000003

6. Kaut KP, DePompei R, Kerr J, Congeni J. Reports of head injury and symptom knowledge among college athletes: implications for assessment and educational intervention. Clin J Sport Med. (2003) 13:213-21. doi: 10.1097/00042752-200307000-00004

7. Kurča E, Sivák Š, Kučera P. Impaired cognitive functions in mild traumatic brain injury patients with normal and pathologic magnetic resonance imaging. Neuroradiology. (2006) 48:661-9. doi: 10.1007/s00234-006-0109-9

8. Lee H, Wintermark M, Gean AD, Ghajar J, Manley GT, Mukherjee P. Focal lesions in acute mild traumatic brain injury and neurocognitive outcome: CT versus 3T MRI. J Neurotrauma. (2008) 25:1049-56. doi: 10.1089/neu.2008.0566

9. Easter JS, Haukoos JS, Meehan WP, Novack V, Edlow JA. Will neuroimaging reveal a severe intracranial injury in this adult with minor head trauma? The rational clinical examination systematic review. JAMA. (2015) 314:267281. doi: 10.1001/jama.2015.16316

\section{AUTHOR CONTRIBUTIONS}

SM and MMF: conceptualization, literature search, and writing. MAFC: literature search. AT: conceptualization, writing, and supervision. JA, J-FK, NL, GM, and SL: writing-review and editing. All authors gave final approval of the manuscript.

\section{FUNDING}

This study was supported by the University and the University Hospital of Liege, the Belgian National Funds for Scientific Research (FRS-FNRS), the European Union's Horizon 2020 Framework Programme for Research and Innovation under Specific Grant Agreement No. 945539 (Human Brain Project SGA3) and No. 686764 (Luminous project), DOCMA project (EU-H2020-MSCA-RISE778234), the European Space Agency (ESA) and the Belgian Federal Science Policy Office (BELSPO) in the framework of the PRODEX Programme, Fondazione Europea di Ricerca Biomedica, the Bial Foundation, the Mind Science Foundation and the European Commission, the fund Generet, the Mind Care International Foundation, and the King Baudouin Foundation.

10. Biagianti B, Stocchetti N, Brambilla P, Van Vleet T. Brain dysfunction underlying prolonged post-concussive syndrome: a systematic review. J Affect Disord. (2020) 262:71-6. doi: 10.1016/j.jad.2019.10.058

11. Eierud C, Craddock RC, Fletcher S, Aulakh M, King-Casas B, Kuehl $\mathrm{D}$, et al. Neuroimaging after mild traumatic brain injury: review and meta-analysis. Neuroimage Clin. (2014) 4:283-94. doi: 10.1016/j.nicl.2013. 12.009

12. Julious SA. Sample size of 12 per group rule of thumb for a pilot study. Pharmaceutical Statist. (2005) 4:287-91. doi: 10.1002/pst.185

13. Ouzzani M, Hammady H, Fedorowicz Z, Elmagarmid A. Rayyana web and mobile app for systematic reviews. Syst Rev. (2016) 5:210. doi: 10.1186/s13643-016-0384-4

14. Datta SGS, Pillai SV, Rao SL, Kovoor JME, Chandramouli BA. Postconcussion syndrome: correlation of neuropsychological deficits, structural lesions on magnetic resonance imaging and symptoms. Neurol India. (2009) 57:594-8. doi: 10.4103/0028-3886.57810

15. Sigurdardottir S, Andelic N, Roe C, Jerstad T, Schanke AK. Post-concussion symptoms after traumatic brain injury at 3 and 12 months post-injury: a prospective study. Brain Inj. (2009) 23:489-97. doi: 10.1080/02699050902926309

16. Zhou Y, Kierans A, Kenul D, Ge Y, Rath J, Reaume J, et al. Mild traumatic brain injury: longitudinal regional brain volume changes. Radiology. (2013) 267:880-90. doi: 10.1148/radiol.13122542

17. Lannsj,ö M., Raininko R, Bustamante M, Von Seth C, Borg J. Brain pathology after mild traumatic brain injury: an exploratory study by repeated magnetic resonance examination. J Rehabil Med. (2013) 45:7218. doi: 10.2340/16501977-1169

18. Killgore WDS, Singh P, Kipman M, Pisner D, Fridman A, Weber M. Gray matter volume and executive functioning correlate with time since injury following mild traumatic brain injury. Neurosci Lett. (2016) 612:23844. doi: 10.1016/j.neulet.2015.12.033

19. Clark AL, Sorg SF, Schiehser DM, Luc N, Bondi MW, Sanderson M, et al. Deep white matter hyperintensities affect verbal memory independent of PTSD symptoms in veterans with mild traumatic brain injury. Brain Inj. (2016) 30:864-71. doi: 10.3109/02699052.2016.1144894

20. Hellstrøm T, Westlye LT, Sigurdardottir S, Brunborg C, Soberg HL, Holthe $\mathrm{O}$, et al. Longitudinal changes in brain morphology from 4 
weeks to 12 months after mild traumatic brain injury: associations with cognitive functions and clinical variables. Brain Inj. (2017) 31:67485. doi: 10.1080/02699052.2017.1283537

21. De Haan S, de Groot JC, Jacobs B, Van der Naalt J. The association between microhaemorrhages and post - traumatic functional outcome in the chronic phase after mild traumatic brain injury. Neuroradiology. (2017) 59:9639. doi: 10.1007/s00234-017-1898-8

22. Chai C, Guo R, Zuo C, Fan L, Liu S, Qian T, et al. Decreased susceptibility of major veins in mild traumatic brain injury is correlated with post-concussive symptoms: a quantitative susceptibility mapping study. Neuroimage Clin. (2017) 15:625-32. doi: 10.1016/j.nicl.2017.06.008

23. Yoo RE, Choi SH, Oh BM, Do Shin S, Lee EJ, Shin DJ, et al. Quantitative dynamic contrast-enhanced MR imaging shows widespread blood-brain barrier disruption in mild traumatic brain injury patients with post-concussion syndrome. Eur Radiol. (2019) 29:1308-17. doi: 10.1007/s00330-018-5656-Z

24. Smits M, Houston GC, Dippel DWJ, Wielopolski PA, Vernooij MW, Koudstaal PJ, et al. Microstructural brain injury in postconcussion syndrome after minor head injury. Neuroradiology. (2011) 53:553-63. doi: 10.1007/s00234-010-0774-6

25. Messé A., Caplain S, Paradot G, Garrigue D, Mineo JF, Ares GS, et al. Diffusion tensor imaging and white matter lesions at the subacute stage in mild traumatic brain injury with persistent neurobehavioral impairment. Hum Brain Mapp. (2011) 32:999-1011. doi: 10.1002/hbm.21092

26. Messé A, Caplain S, Pélégrini-Issac M, Blancho S, Montreuil M, Lévy $\mathrm{R}$, et al. Structural integrity and postconcussion syndrome in mild traumatic brain injury patients. Brain Imaging Behav. (2012) 6:28392. doi: 10.1007/s11682-012-9159-2

27. Lange RT, Iverson GL, Brubacher JR, Mädler B, Heran MK. Diffusion tensor imaging findings are not strongly associated with postconcussional disorder 2 months following mild traumatic brain injury. J Head Trauma Rehabil. (2012) 27:188-98. doi: 10.1097/HTR.0b013e318217f0ad

28. Yeh PH, Wang B, Oakes TR, French LM, Pan H, Graner J, et al. Postconcussional disorder and PTSD symptoms of military-related traumatic brain injury associated with compromised neurocircuitry. Hum Brain Mapp. (2014) 35:2652-73. doi: 10.1002/hbm.22358

29. Ilvesmäki T, Luoto TM, Hakulinen U, Brander A, Ryymin P, Eskola H, et al. Acute mild traumatic brain injury is not associated with white matter change on diffusion tensor imaging. Brain. (2014) 137:187682. doi: 10.1093/brain/awu095

30. Wäljas M, Lange RT, Hakulinen U, Huhtala H, Dastidar P, Hartikainen K, et al. Biopsychosocial outcome after uncomplicated mild traumatic brain injury. J Neurotrauma. (2014) 31:108-24. doi: 10.1089/neu.2013.2941

31. Petrie EC, Cross DJ, Yarnykh VL, Richards T, Martin NM. Pagulayan k, et al. Neuroimaging, behavioral, and psychological sequelae of repetitive combined blast/impact mild traumatic brain injury in Iraq and Afghanistan war veterans. J Neurotrauma. (2014) 31:425-36. doi: 10.1089/neu.2013.2952

32. Panenka WJ, Lange RT, Bouix S, Shewchuk JR, Heran MKS, Brubacher JR, et al. Neuropsychological outcome and diffusion tensor imaging in complicated versus uncomplicated mild traumatic brain injury. PLoS ONE. (2015) 10:122746. doi: 10.1371/journal.pone.0122746

33. Davenport ND, Lim KO, Sponheim SR. Personality and neuroimaging measures differentiate PTSD from MTBI in veterans. Brain Imaging Behav. (2015) 9:472-83. doi: 10.1007/s11682-015-9371-y

34. Lange RT, Panenka WJ, Shewchuk JR, Heran MKS, Brubacher JR, Bioux S, et al. Diffusion tensor imaging findings and postconcussion symptom reporting six weeks following mild traumatic brain injury. Arch Clin Neuropsychol. (2015) 30:7-25. doi: 10.1093/arclin/acu060

35. Wäljas M, Iverson GL, Lange RT, Hakulinen U, Dastidar P, Huhtala H, et al. A prospective biopsychosocial study of the persistent post-concussion symptoms following mild traumatic brain injury. J Neurotrauma. (2015) 32:534-47. doi: 10.1089/neu.2014.3339

36. Delano-Wood L, Bangen KJ, Sorg SF, Clark AL, Schiehser DM, Luc N, et al. Brainstem white matter integrity is related to loss of consciousness and postconcussive symptomatology in veterans with chronic mild to moderate traumatic brain injury. Brain Imaging Behav. (2015) 9:50012. doi: $10.1007 / \mathrm{s} 11682-015-9432-2$

37. Miller DR, Hayes JP, Lafleche G, Salat DH, Verfaellie M. White matter abnormalities are associated with chronic postconcussion symptoms in blast-related mild traumatic brain injury. Hum Brain Mapp. (2016) 37:2209. doi: 10.1002/hbm.23022

38. Meier TB, Bergamino M, Bellgowan PSF, Teague TK, Ling JM, Jeromin $A$, et al. Longitudinal assessment of white matter abnormalities following sports-related concussion. Hum Brain Mapp. (2016) 37:833-45. doi: 10.1002/hbm.23072

39. Lancaster MA, Olson DV, McCrea MA, Nelson LD, LaRoche AA, Muftuler LT. Acute white matter changes following sport-related concussion: a serial diffusion tensor and diffusion kurtosis tensor imaging study. Hum Brain Mapp. (2016) 37:3821-34. doi: 10.1002/hbm.23278

40. Astafiev SV, Zinn KL, Shulman GL, Corbetta M. Exploring the physiological correlates of chronic mild traumatic brain injury symptoms. Neuroimage Clin. (2016) 11:10-9. doi: 10.1016/j.nicl.2016.01.004

41. Thomas AW, Watts R, Filippi CG, Nickerson JP, Andrews T, Lieberman $\mathrm{G}$, et al. Dynamic changes in diffusion measures improve sensitivity in identifying patients with mild traumatic brain injury. PLoS ONE. (2017) 12:178360. doi: 10.1371/journal.pone.0178360

42. Yeh PH, Koay CG, Wang B, Morissette J, Sham E, Senseney J, et al. Compromised neurocircuitry in chronic blast-related mild traumatic brain injury. Hum Brain Mapp. (2017) 38:352-69. doi: 10.1002/hbm.23365

43. Næss-Schmidt ET, Blicher JU, Eskildsen SF, Tietze A, Hansen B, Stubbs PW, et al. Microstructural changes in the thalamus after mild traumatic brain injury: a longitudinal diffusion and mean kurtosis tensor MRI study. Brain Inj. (2017) 31:230-6. doi: 10.1080/02699052.2016.1229034

44. Rangaprakash D, Deshpande G, Daniel TA, Goodman AM, Robinson JL, Salibi N, et al. Compromised hippocampus-striatum pathway as a potential imaging biomarker of mild-traumatic brain injury and posttraumatic stress disorder. Hum Brain Mapp. (2017) 38:2843-64. doi: 10.1002/hbm.23551

45. Klimova A, Korgaonkar MS, Whitford T, Bryant RA. Diffusion tensor imaging analysis of mild traumatic brain injury and posttraumatic stress disorder. Biol Psychiatry Cogn Neurosci Neuroimaging. (2019) 4:8190. doi: 10.1016/j.bpsc.2018.10.004

46. Lancaster MA, Meier TB, Olson DV, McCrea MA, Nelson LD, Muftuler LT. Chronic differences in white matter integrity following sport-related concussion as measured by diffusion MRI: 6-Month follow-up. Hum Brain Mapp. (2018) 39:4276-89. doi: 10.1002/hbm.24245

47. Yin B, Li DD, Huang $\mathrm{H}, \mathrm{Gu} \mathrm{CH}$, Bai GH, Hu LX, et al. Longitudinal changes in diffusion tensor imaging following mild traumatic brain injury and correlation with outcome. Front Neural Circuits. (2019) 13:28. doi: 10.3389/fncir.2019.00028

48. Smits M, Dippel DWJ, Houston GC, Wielopolski PA, Koudstaal PJ, Hunink MGM, et al. Postconcussion syndrome after minor head injury: brain activation of working memory and attention. Hum Brain Mapp. (2009) 30:2789-803. doi: 10.1002/hbm.20709

49. Tang L, Ge Y, Sodickson DK, Miles L, Zhou Y, Reaume J, et al. Thalamic resting-state functional networks: disruption in patients with mild traumatic brain injury. Radiology. (2011) 260:831-40. doi: 10.1148/radiol.11110014

50. Gosselin N, Bottari C, Chen JK, Petrides M, Tinawi S, De Guise E, et al. Electrophysiology and functional MRI in post-acute mild traumatic brain injury. J Neurotrauma. (2011) 28:329-41. doi: 10.1089/neu.2010.1493

51. Stevens MC, Lovejoy D, Kim J, Oakes H, Kureshi I, Witt ST. Multiple resting state network functional connectivity abnormalities in mild traumatic brain injury. Brain Imaging Behav. (2012) 6:293-318. doi: 10.1007/s11682-012-9157-4

52. Terry DP, Faraco CC, Smith D, Diddams MJ, Puente AN, Miller LS. Lack of long-term FMRI differences after multiple sports-related concussions. Brain Inj. (2012) 26:1684-96. doi: 10.3109/02699052.2012.722259

53. Zhou Y, Milham MP, Lui YW, Miles L, Reaume J, Sodickson DK, et al. Defaultmode network disruption in mild traumatic brain injury. Radiology. (2012) 265:882-92. doi: 10.1148/radiol.12120748

54. Messé A., Caplain S, Pélégrini-Issac M, Blancho S, Lévy R, Aghakhani $\mathrm{N}$, et al. Specific and evolving resting-state network alterations in postconcussion syndrome following mild traumatic brain injury. PLoS ONE. (2013) 8:e65470. doi: 10.1371/journal.pone.0065470

55. Mutch WAC, Ellis MJ, Graham MR, Wourms V, Raban R, Fisher JA, et al. Brain MRI CO2 stress testing: a pilot study in patients with concussion. PLoS ONE. (2014) 9:102181. doi: 10.1371/journal.pone.0102181

56. Sours C, Chen H, Roys S, Zhuo J, Varshney A, Gullapalli RP. Investigation of multiple frequency ranges using discrete wavelet decomposition of 
resting-state functional connectivity in mild traumatic brain injury patients. Brain Connect. (2015) 5:442-50. doi: 10.1089/brain.2014.0333

57. Sours C, Zhuo J, Roys S, Shanmuganathan K, Gullapalli RP. Disruptions in resting state functional connectivity and cerebral blood flow in mild traumatic brain injury patients. PLOS ONE. (2015) 10:e0134019. doi: 10.1371/journal.pone.0134019

58. Sours C, George EO, Zhuo J, Roys S, Gullapalli RP. Hyper-connectivity of the thalamus during early stages following mild traumatic brain injury. Brain Imaging Behav. (2015) 9:550-63. doi: 10.1007/s11682-015-9424-2

59. Iraji A, Benson RR, Welch RD, O'Neil BJ, Woodard JL, Ayaz SI, et al. Resting state functional connectivity in mild traumatic brain injury at the acute stage: independent component and seed-based analyses. J Neurotrauma. (2015) 32:1031-45. doi: 10.1089/neu.2014.3610

60. Koski L, Kolivakis T, Yu C, Chen JK, Delaney S, Ptito A. Noninvasive brain stimulation for persistent postconcussion symptoms in mild traumatic brain injury. J Neurotrauma. (2015) 32:38-44. doi: 10.1089/neu.20 14.3449

61. Van der Horn HJ, Liemburg EJ, Scheenen ME, De Koning ME, Spikman JM, Van der Naalt J. Post-concussive complaints after mild traumatic brain injury associated with altered brain networks during working memory performance. Brain Imaging Behav. (2016) 10:1243-53. doi: 10.1007/s11682-015-9489-y

62. Banks SD, Coronado RA, Clemons LR, Abraham CM, Pruthi S, Conrad BN, et al. Thalamic functional connectivity in mild traumatic brain injury: longitudinal associations with patient-reported outcomes and neuropsychological tests. Arch Phys Med Rehabil. (2016) 97:1254-61. doi: 10.1016/j.apmr.2016.03.013

63. Palacios EM, Yuh EL, Chang YS, Yue JK, Schnyer DM, Okonkwo DO, et al. Resting-state functional connectivity alterations associated with six-month outcomes in mild traumatic brain injury. J Neurotrauma. (2017) 34:154657. doi: 10.1089/neu.2016.4752

64. Zhou Y. Small world properties changes in mild traumatic brain injury. J Magn Reson Imaging. (2017) 46:518-27. doi: 10.1002/jmri.25548

65. Kaushal M, España LY, Nencka AS, Wang Y, Nelson LD, McCrea $\mathrm{MA}$, et al. Resting-state functional connectivity after concussion is associated with clinical recovery. Hum Brain Mapp. (2019) 40:121120. doi: $10.1002 / \mathrm{hbm} .24440$

66. Chong CD, Wang L, Wang K, Traub S, Li J. Homotopic region connectivity during concussion recovery: a longitudinal fMRI study. PLoS ONE. (2019) 14:e0221892. doi: 10.1371/journal.pone.0221892

67. Hou W, Rhodes SC, Jiang L, Roys S, Zhuo J, JaJa J, et al. Dynamic functional network analysis in mild traumatic brain injury. Brain Connect. (2019) 9:47587. doi: 10.1089/brain.2018.0629

68. Manning KY, Llera A, Dekaban GA, Bartha R, Barreira C, Brown A, et al. Linked MRI signatures of the brain's acute and persistent response to concussion in female varsity rugby players. Neuroimage Clin. (2019) 21:101627. doi: 10.1016/j.nicl.2018.101627

69. Gosselin N, Bottari C, Chen JK, Huntgeburth SC, De Beaumont L, Petrides $\mathrm{ML}$, et al. Evaluating the cognitive consequences of mild traumatic brain injury and concussion by using electrophysiology. Neurosurg Focus. (2012) 33:E7:1-7. doi: 10.3171/2012.10.FOCUS12253

70. Larson MJ, Clayson PE, Farrer TJ. Performance monitoring and cognitive control in individuals with mild traumatic brain injury. J Int Neuropsychol Soc. (2012) 18:323-33. doi: 10.1017/S1355617711001779

71. Huang MX, Nichols S, Baker DG, Robb A, Angeles A, Yurgil KA, et al. Singlesubject-based whole-brain MEG slow-wave imaging approach for detecting abnormality in patients with mild traumatic brain injury. Neuroimage Clin. (2014) 5:109-19. doi: 10.1016/j.nicl.2014.06.004

72. Robb Swan A, Nichols S, Drake A, Angeles AM, Diwakar M, Song T, et al. Magnetoencephalography slow-wave detection in patients with mild traumatic brain injury and ongoing symptoms correlated with long-term neuropsychological outcome. J Neurotrauma. (2015) 32:151021. doi: $10.1089 /$ neu.2014.3654

73. Vakorin VA, Doesburg SM, Da Costa L, Jetly R, Pang EW, Taylor MJ. Detecting mild traumatic brain injury using resting state magnetoencephalographic connectivity. PLoS Comput Biol. (2016) 12:1-24. doi: 10.1371/journal.pcbi.1004914
74. Huang MX, Harrington DL, Robb A, Quinto AA, Nichols S, Drake A, et al. Resting-state magnetoencephalography reveals different patterns of aberrant functional connectivity in combat-related mild traumatic brain injury. $J$ Neurotrauma. (2017) 34:1412-26. doi: 10.1089/neu.2016.4581

75. Lewine JD, Plis S, Ulloa A, Williams C, Spitz M, Foley J, et al. Quantitative EEG biomarkers for mild traumatic brain injury. J Clin Neurophysiol. (2019) 36:298-305. doi: 10.1097/WNP.0000000000000588

76. Ruiter KI, Boshra R, Doughty M, Noseworthy M, Connolly JF. Disruption of function: neurophysiological markers of cognitive deficits in retired football players. Clin Neurophysiol. (2019) 130:111-21. doi: 10.1016/j.clinph.2018.10.013

77. Egner $\mathrm{T}$, Hirsch J. The neural correlates and functional integration of cognitive control in a stroop task. Neuroimage. (2005) 24:53947. doi: 10.1016/j.neuroimage.2004.09.007

78. Motzkin JC, Philippi CL, Wolf RC, Baskaya MK, Koenigs M. Ventromedial prefrontal cortex is critical for the regulation of amygdala activity in humans. Biol Psychiatry. (2015) 77:276-84. doi: 10.1016/j.biopsych.2014.02.014

79. Owen AM, McMillan KM, Laird AR, Bullmore E. N-back working memory paradigm: a meta-analysis of normative functional neuroimaging studies. Hum Brain Mapp. (2005) 25:46-59. doi: 10.1002/hbm.20131

80. Euston DR, Gruber AJ, McNaughton BL. The role of medial prefrontal cortex in memory and decision making. Neuron. (2012) 76:1057-70. doi: 10.1016/j.neuron.2012.12.002

81. Van der Werf YD, Witter MP, Uylings HBM, Jolles J. Neuropsychology of infarctions in the thalamus: a review. Neuropsychologia. (2000) 38:61327. doi: 10.1016/S0028-3932(99)00104-9

82. Vanhaudenhuyse A, Demertzi A, Schabus $\mathrm{M}$, Noirhomme Q, Bredart S, Boly $M$, et al. Two distinct neuronal networks mediate the awareness of environment and of self. J Cogn Neurosci. (2011) 23:570-8. doi: 10.1162/jocn.2010.21488

83. Di X, Biswal BB. Modulatory interactions between the default mode network and task positive networks in resting-state. PeerJ. (2014) 2:e367. doi: 10.7717/peerj.367

84. Seeley WW, Menon V, Schatzberg AF, Keller J, Glover GH, Kenna $\mathrm{H}$, et al. Dissociable intrinsic connectivity networks for salience processing and executive control. J Neurosci. (2007) 27:2349-56. doi: 10.1523/JNEUROSCI.5587-0.2007

85. Weiner KS, Grill-Spector K. Sparsely-distributed organization of face and limb activations in human ventral temporal cortex. Neuroimage. (2010) 52:1559-73. doi: 10.1016/j.neuroimage.2010..262

86. Huang MX, Theilmann RJ, Robb A, Angeles A, Nichols S, Drake A, et al. Integrated imaging approach with MEG and DTI to detect mild traumatic brain injury in military and civilian patients. J Neurotrauma. (2009) 26:121326. doi: $10.1089 /$ neu. 200.0672

87. Hannawi Y, Stevens RD. Mapping the connectome following traumatic brain injury. Curr Neurol Neurosci Rep. (2016) 16:44. doi: $10.1007 /$ s11910-016-0642-9

Conflict of Interest: The authors declare that the research was conducted in the absence of any commercial or financial relationships that could be construed as a potential conflict of interest.

Publisher's Note: All claims expressed in this article are solely those of the authors and do not necessarily represent those of their affiliated organizations, or those of the publisher, the editors and the reviewers. Any product that may be evaluated in this article, or claim that may be made by its manufacturer, is not guaranteed or endorsed by the publisher.

Copyright $\odot 2021$ Mortaheb, Filippini, Kaux, Annen, Lejeune, Martens, Calderón, Laureys and Thibaut. This is an open-access article distributed under the terms of the Creative Commons Attribution License (CC BY). The use, distribution or reproduction in other forums is permitted, provided the original author(s) and the copyright owner(s) are credited and that the original publication in this journal is cited, in accordance with accepted academic practice. No use, distribution or reproduction is permitted which does not comply with these terms. 